\title{
DIU con Levonorgestrel comparable a la histerectomía para tratar la hipermenorrea
}

\section{Objetivo}

Comparar resultados del tratamiento de la hipermenorrea con Dispositivo Intrauterino (DIU) con levonorgestrel vs. histerectomía en relación a la calidad de vida y costos.

\section{Diseño}

Ensayo clínico aleatorizado y controlado de 5 años de seguimiento

\section{Lugar}

Estudio multicéntrico realizado en 5 hospitales universitarios en Finlandia.

\section{Pacientes}

Mujeres premenopáusicas $(\mathrm{n}=236)$ con hipermenorrea.

\section{Intervención}

Las pacientes fueron aleatorizados a recibir DIU con levonorgestrel $(n=119)$ o histerectomía $(n=117)$.

\section{Medición de resultados principales}

La calidad de vida fue estimada por los índices obtenidos a través del 5-Dimensional EuroQol (EQ-5D). Además se realizaron otras mediciones de bienestar psicosocial (ansiedad, depresión y función sexual), satisfacción y costos.

\section{Resultados principales}

Los resultados de los dos grupos son similares en lo que respecta a calidad de vida, satisfacción y bienestar psicofísico, con una alta tasa de aceptación para ambos (tabla). Los costos son menores en el grupo de usuarias de DIU. El 52\% de ellas discontinuó el méto- do y la mayoría (88\%) necesitó tratamiento quirúrgico, lo que aumentó los costos pero sin llegar a superar al grupo histerectomía.

\begin{tabular}{|c|c|c|c|}
\hline & \multicolumn{2}{|c|}{ Gambios de puntaje en 5 años (IC 95\%) } & \multirow[t]{2}{*}{$\mathbf{p}$} \\
\hline Mediciones & DIU (n=117) & Histerectomía ( $n=115)$ & \\
\hline EQ-5D (rango de escala: $0-1)^{*}$ & $0.08(0.03$ a 0.13$)$ & $0.10(0.05$ a 0.15$)$ & NS \\
\hline Rand-36 (rango de escala: $0-100)^{*}$ & & & \\
\hline -Salud general & $3.6(0.1$ a 7.1$)$ & $4.4(1.0$ a 7.8$)$ & NS \\
\hline -Funcionamiento físico & $-1.4(-5.1$ a 2.2$)$ & $-2.0(-5.6$ a 1.6$)$ & NS \\
\hline -Bienestar emocional & $8.4(4.7$ a 12.2$)$ & $8.1(4.9$ a 11.4$)$ & NS \\
\hline -Funcionamiento social & $8.7(4.1$ a 13.3$)$ & $9.0(4.5$ a 13.6$)$ & NS \\
\hline -Energía & $9.4(5.3$ a 13.6$)$ & $10.0(5.8$ a 14.1$)$ & NS \\
\hline -Dolor & $12.8(7.9$ a 17.7$)$ & $13.4(7.7$ a 19.1$)$ & NS \\
\hline -Rol en funcionamiento & & & \\
\hline .físico & $8.9(1.3$ a 16.4$)$ & $10.8(2.4$ a 19.2$)$ & NS \\
\hline emocional & $16.2(8.4$ a 24.0$)$ & $12.9(4.0$ a 21.8$)$ & NS \\
\hline $\begin{array}{l}\text { Salud general (escala VAS; } \\
\text { rango de escala: } 0-100)^{*}\end{array}$ & $0.4(-5.1$ a 6.0$)$ & $4.4(0.2$ a 8.6$)$ & NS \\
\hline Ansiedad (STAI [rango de escala: 20-80]) & $-2.4(-3.5$ a -1.3$)$ & $-1.9(-3.1$ a -0.8$)$ & NS \\
\hline Depresión (BDI [rango de escala: 0-39]) & $-1.2(-2.0$ a -0.3$)$ & $-1.4(-2.0$ a -0.6$)$ & NS \\
\hline Sexualidad (MSS) & & & \\
\hline -Satisfacción (rango: 5-35)\# & $-0.7(-1.8$ a 0.3$)$ & $0.2(-0.9$ a 1.4$)$ & NS \\
\hline -Problemas (rango: 2-14) & $-0.02(-0.6$ a 0.5$)$ & $-0.04(-0.6$ a 0.5$)$ & NS \\
\hline -Satisfacción con la pareja (rango: 3-21)\# & $-0.7(-1.2$ a -0.2$)$ & $-0.4(-1.0$ a 0.3$)$ & NS \\
\hline
\end{tabular}

Mayores puntajes indican: *mayor calidad de vida relacionada a la salud, más síntomas o problemas, \# más satisfacción, NS:no significativo.

\section{Conclusiones}

EI DIU con levonorgestrel es una opción válida dentro de los tratamientos de las pacientes con hipermenorrea. Mejora la calidad de vida de estas pacientes a un costo relativamente bajo y en un grupo de ellas puede evitar la histerectomía.

Fuente de Financiamiento: Academy of Finland, STAKES y Research Funds of the University Hospitals. Los DIU fueron provistos por Schering Co, Finland.

\section{Comentario}

Al diagnóstico de sangrado uterino disfuncional se llega al descartar otras causas de sangrado uterino anormal (miomas, pólipos, carcinomas, alteraciones de la coagulación, trastornos endocrinológicos, enfermedades sistémicas, etc). Las pacientes del estudio no deseaban embarazo ni presentaban dismenorrea intensa, tenían un examen físico normal, Pap negativo, ecografia, determinaciones hormonales y coagulograma normales. La menometrorragia es el síntoma más común de presentación ${ }^{1-2}$ pero es la hipermenorrea la que las puede llevar a la anemia. Los tratamientos disponibles son: tratamiento médico (estrógenos, progesterona, antifibrinolíticos, danazol, gestrinona, análogos GnRh, AINE, y hierro); DIU con levonorgestrel y tratamientos quirúrgicos conservadores como la ablación endometral y radicales como la histerectomía. En general se plantea inicialmente el tratamiento médico. EI DIU con levonorgestrel fue desarrollado como método anticonceptivo y posteriormente aplicado al tratamiento del sangrado anormal al observar que en las usuarias se lograba una disminución importante del sangrado menstrual y que muchas de ellas llegaban incluso a la amenorrea. ${ }^{3}$ Sin embargo conserva los efectos adversos comunes a los DIU a los que suma los derivados de la acción hormonal. En este estudio se comparan dos tratamientos para la hipermenorrea: DIU con levonorgestrel vs histerectomía y se analizan los resultados en cuanto a la calidad de vida, satisfacción, otras mediciones de bienestar psicofísico (ansiedad, depresión y función sexual) y costos. Luego de un seguimiento de 5 años con una baja tasa de pérdida de seguimiento $(1 \%)$ ambos grupos presentaron una alta tasa de satisfacción $(>90 \%)$. Se obtuvieron semejantes resultados en ambos grupos para los parámetros relacionados con la calidad de vida y bienestar psicofísico pero con menores costos con DIU. Sin embargo el $52 \%$ de las usuarias de DIU discontinuó el método (por metrorragia intermenstrual, hipermenorrea y síntomas hormonales) y necesitó otro tratamiento incluido el quirúrgico lo que aumentó los costos pero sin superar los del grupo histerectomía. Hay dos situaciones que llaman la atención. Por un lado la alta tasa de complicaciones intra y post quirúrgica reportada en relación a algunos reportes ${ }^{4},{ }^{5}$ lo que aumenta los costos. Por otro lado la alta tasa de discontinuación del DIU (52\%), lo que implica segundas intervenciones, pero esto no se ve reflejado negativamente en los resultados de satisfacción y calidad de vida como si ocurre en otras publicaciones.

Concluyen los autores que el DIU con levonorgestrel es una opción para el tratamiento de las pacientes con hipermenorrea, que mejora la calidad de vida de estas mujeres a relativo bajo costo, y que en muchos casos puede evitar la histerectomía.

Conclusión del comentador: La correcta selección de pacientes es la mejor forma de evitar la insatisfacción con los tratamientos y el aumento de los costos por la necesidad de segundos tratamientos. Nunca se deben generar falsas expectativas y promesas de resultados. La elección del método a emplear se debe tomar en conjunto con la paciente luego de exponer pro y contras de cada uno y evaluar las necesidades de la paciente en forma individual.

\section{Dr. Santiago Gil [ Servicio de Ginecología. Hospital Italiano de Buenos Aires. ]}

Gil S. DIU con Levonorgestrel comparable a la histerectomía para tratar la hipermenorrea . Evid. actual. práct. ambul.2004;7:135. Comentado de: Clinical outcomes and costs with the Levonorgestrel-Releasing Intrauterine System or Hysterectomy for the treatment of menorrhagia. Hurskainen R, Teperi J, Rissanen P. JAMA 2004;291:1456-63.

\section{Referencias}

1.Vuorma S, Teperi J, Hurskainen R, Keskimäki I, Kujansuu E. Hysterectomy trends in Finland in 1987-1995-a register based analysis. Acta Obstet Gynecol Scand. 1998;77:770-776. 2.Stirrat GM. Choice of treatment for menorrhagia. Lancet. 1999;353:2175-2176.

3.Lähteenmäki P, Haukkamaa M, Puolakka J, et al. Open randomised study of use of levonorgestrel releasing intrauterine system as alternative to hysterectomy.BMJ.1998;316:1122-1126. 4.Munro MG, Deprest J. Laparoscopic hysterectomy: does it work? Clin Obstet Gynecol. 1995:38:401-425.

5.Härkki-Siren P, Sjöberg J, Mäkinen J, et al. Finnish national register of laparoscopic hysterectomies. Am J Obstet Gynecol. 1997;176:118-122. 